\title{
Functional Inequalities Associated with Cauchy Additive Functional Equation in Non-Archimedean Spaces
}

\author{
A. Ebadian, ${ }^{1}$ N. Ghobadipour, ${ }^{1}$ Th. M. Rassias, ${ }^{2}$ \\ and M. Eshaghi Gordji $3,4,5$ \\ ${ }^{1}$ Department of Mathematics, Urmia University, Urmia, Iran \\ ${ }^{2}$ Department of Mathematics, National Technical University of Athens, 15780 Zografou, Greece \\ ${ }^{3}$ Department of Mathematics, Semnan University, P. O. Box 35195-363, Iran \\ ${ }^{4}$ Research Group of Nonlinear Analysis and Applications (RGNAA), Semnan University, Iran \\ ${ }^{5}$ Center of Excellence in Nonlinear Analysis and Applications (CENAA), Semnan University, Iran
}

Correspondence should be addressed to M. Eshaghi Gordji, madjid.eshaghi@gmail.com

Received 30 October 2010; Accepted 19 January 2011

Academic Editor: Rigoberto Medina

Copyright (C) 2011 A. Ebadian et al. This is an open access article distributed under the Creative Commons Attribution License, which permits unrestricted use, distribution, and reproduction in any medium, provided the original work is properly cited.

We investigate the generalized Hyers-Ulam stability of the functional inequalities $\| f((x+y+z) / 4)+$ $f((3 x-y-4 z) / 4)+f((4 x+3 z) / 4)\|\leq\| 2 f(x) \|$ and $\|f((y-x) / 3)+f((x-3 z) / 3)+f((3 x+3 z-y) / 3)\| \leq$ $\|f(x)\|$ in non-Archimedean normed spaces in the spirit of the Th. M. Rassias stability approach.

\section{Introduction}

Ulam [1] gave a talk before the Mathematics Club of the University of Wisconsin in which he discussed a number of unsolved problems. Among these was the following question concerning the stability of homomorphisms.

Let $\left(G_{1}, \cdot\right)$ be a group and let $\left(G_{2}, *\right)$ be a metric group with the metric $d(\cdot, \cdot)$. Given $\epsilon>0$, does there exist a $\delta>0$, such that if a mapping $h: G_{1} \rightarrow G_{2}$ satisfies the inequality $d(h(x$. $y), h(x) * h(y))<\delta$ for all $x, y \in G_{1}$, then there exists a homomorphism $H: G_{1} \rightarrow G_{2}$ with $d(h(x), H(x))<\epsilon$ for all $x \in G_{1}$ ?

In other words, under what condition does there exist a homomorphism near an approximate homomorphism? The concept of stability for functional equation arises when we replace the functional equation by an inequality which acts as a perturbation of the equation. In 1941, Hyers [2] gave the first affirmative answer to the question of Ulam for Banach spaces. Let $f: E \rightarrow E^{\prime}$ be a mapping between Banach spaces such that

$$
\|f(x+y)-f(x)-f(y)\| \leq \delta
$$


for all $x, y \in E$, and for some $\delta>0$. Then there exists a unique additive mapping $T: E \rightarrow E^{\prime}$ such that

$$
\|f(x)-T(x)\| \leq \delta
$$

for all $x \in E$. Moreover, if $f(t x)$ is continuous in $t \in \mathbb{R}$ for each fixed $x \in E$, then $T$ is linear. In 1978, Rassias [3] proved the following theorem.

Theorem 1.1. Let $f: E \rightarrow E^{\prime}$ be a mapping from a normed vector space $E$ into a Banach space $E^{\prime}$ subject to the inequality

$$
\|f(x+y)-f(x)-f(y)\| \leq \epsilon\left(\|x\|^{p}+\|y\|^{p}\right)
$$

for all $x, y \in E$, where $\epsilon$ and $p$ are constants with $\epsilon>0$ and $p<1$. Then there exists a unique additive mapping $T: E \rightarrow E^{\prime}$ such that

$$
\|f(x)-T(x)\| \leq \frac{2 \epsilon}{2-2^{p}}\|x\|^{p}
$$

for all $x \in E$. If $p<0$ then inequality (1.3) holds for all $x, y \neq 0$, and (1.4) for $x \neq 0$. Also, if the function $t \mapsto f(t x)$ from $\mathbb{R}$ into $E^{\prime}$ is continuous in real $t$ for each fixed $x \in E$, then $T$ is linear.

In 1991, Gajda [4] answered the question for the case $p>1$, which was raised by Rassias. This new concept is known as Hyers-Ulam-Rassias stability of functional equations. The reader is referred to [5-13] for a number of results in this domain of research. [14].

In 1994, a generalization of the Rassias theorem was obtained by Găvruţa as follows

Suppose $(G,+)$ is an abelian group, $E$ is a Banach space, and that the so-called admissible control function $\varphi: G \times G \rightarrow \mathbb{R}$ satisfies

$$
\tilde{\varphi}(x, y):=2^{-1} \sum_{n=0}^{\infty} 2^{-n} \varphi\left(2^{n} x, 2^{n} y\right)<\infty
$$

for all $x, y \in G$. If $f: G \rightarrow E$ is a mapping with

$$
\|f(x+y)-f(x)-f(y)\| \leq \varphi(x, y)
$$

for all $x, y \in G$, then there exists a unique mapping $T: G \rightarrow E$ such that $T(x+y)=T(x)+T(y)$ and $\|f(x)-T(x)\| \leq \tilde{\varphi}(x, x)$ for all $x, y \in G$.

During the last decades, several stability problems of functional equations have been investigated by a number of mathematicians, see [15-17] and references therein for more detailed information.

By a non-Archimedean field we mean a field $K$ equipped with a function (valuation) $|\cdot|$ from $K$ into $[0,1)$ such that $|r|=0$ if and only if $r=0,|r s|=|r||s|$, and $|r+s| \leq \max \{|r|,|s|\}$ for all $r, s \in K$. Clearly $|1|=|-1|=1$ and $|n| \leq 1$ for all $n \in \mathbb{N}$. 
Let $X$ be a vector space over a scalar field $\mathbb{K}$ with a non-Archimedean nontrivial valuation $|\cdot|$. A function $\|\cdot\|: X \rightarrow \mathbb{R}$ is a non-Archimedean norm (valuation) if it satisfies the following conditions:

(i) $\|x\|=0$ if and only if $x=0$;

(ii) $\|r x\|=|r|\|x\|(r \in \mathbb{K}, x \in X)$;

(iii) the strong triangle inequality (ultrametric), namely,

$$
\|x+y\| \leq \max \{\|x\|,\|y\|\} \quad(x, y \in X) .
$$

Then $(X,\|\cdot\|)$ is called a non-Archimedean space. Due to the fact that

$$
\left\|x_{n}-x_{m}\right\| \leq \max \left\{\left\|x_{j+1}-x_{j}\right\|: m \leq j \leq n-1\right\} \quad(n>m),
$$

a sequence $\left\{x_{n}\right\}$ is Cauchy if and only if $\left\{x_{n+1}-x_{n}\right\}$ converges to zero in a non-Archimedean space. By a complete non-Archimedean space we mean one in which every Cauchy sequence is convergent (see [18-22]).

Gilányi [23] and Rätz [24] showed that if $f$ satisfies the functional inequality

$$
\left\|2 f(x)+2 f(y)-f\left(x y^{-1}\right)\right\| \leq f(x y),
$$

then $f$ satisfies the Jordan-von Neumann functional equation

$$
2 f(x)+2 f(y)=f(x y)+f\left(x y^{-1}\right) .
$$

Gilányi [23] and Fechner [25] proved the generalized Hyers-Ulam stability of the functional inequality (1.3).

Cho and Kim [26] proved the generalized Hyers-Ulam stability of the following functional inequalities:

$$
\begin{gathered}
\left\|f\left(\frac{x-y}{2}-z\right)+f(y)+2 f(z)\right\| \leq\left\|f\left(\frac{x+y}{2}+z\right)\right\|+\varphi(x, y, z), \\
\|f(x)+f(y)+2 f(z)\| \leq\left\|2 f\left(\frac{x+y}{2}+z\right)\right\|+\varphi(x, y, z),
\end{gathered}
$$

which are associated with Jordan-von Neumann-type Cauchy-Jensen additive functional equations.

Now, we consider the following functional inequality:

$$
\begin{gathered}
\left\|f\left(\frac{x+y+z}{4}\right)+f\left(\frac{3 x-y-4 z}{4}\right)+f\left(\frac{4 x+3 z}{4}\right)\right\| \leq\|2 f(x)\|, \\
\left\|f\left(\frac{y-x}{3}\right)+f\left(\frac{x-3 z}{3}\right)+f\left(\frac{3 x+3 z-y}{3}\right)\right\| \leq\|f(x)\|,
\end{gathered}
$$

which is associated with Cauchy additive functional equation. 
The purpose of this paper is to prove that if $f$ satisfies the inequalities (1.12) and (1.13), which satisfies certain conditions, then $f$ is Cauchy additive, and thus we prove the generalized Hyers-Ulam stability of the functional inequalities (1.12) and (1.13) in nonArchimedean normed spaces.

\section{Stability of Functional Inequality (1.12)}

In this section, we prove the generalized Hyers-Ulam stability of the functional inequality (1.12). Throughout this section, we assume that $S$ is an additive semigroup and $X$ is a complete non-Archimedean space.

We need the following lemma in the main results.

Lemma 2.1. Let $f: S \rightarrow X$ be a mapping such that

$$
\left\|f\left(\frac{x+y+z}{4}\right)+f\left(\frac{3 x-y-4 z}{4}\right)+f\left(\frac{4 x+3 z}{4}\right)\right\| \leq\|2 f(x)\|
$$

for all $x, y, z \in S$. If $|3|>|2|$, then the mapping $f$ is Cauchy additive.

Proof. Letting $x=y=z=0$ in (2.1), we get $|3|\|f(0)\| \leq|2|\|f(0)\|$. So, $f(0)=0$. Letting $x=z=0$ and replacing $y$ by $4 y$ in (2.1), we get $\|f(y)+f(-y)\| \leq|2|\|f(0)\|$ for all $y \in S$. So, $f(-y)=-f(y)$ for all $y \in S$. Setting $x=0$ in (2.1), we obtain

$$
\left\|f\left(\frac{y+z}{4}\right)+f\left(\frac{-y-4 z}{4}\right)+f\left(\frac{3 z}{4}\right)\right\| \leq|2|\|f(0)\| .
$$

So,

$$
f\left(\frac{y+z}{4}\right)+f\left(\frac{-y-4 z}{4}\right)+f\left(\frac{3 z}{4}\right)=0
$$

for all $y, z \in S$. Replacing $y$ by $2 z$ in (2.3), we get

$$
2 f\left(\frac{3 z}{4}\right)=f\left(\frac{3 z}{2}\right)
$$

for all $z \in S$. Using (2.4), we obtain $f(2 z)=2 f(z)$ and $f(4 z)=4 f(z)$ for all $z \in S$. Letting $x=0, w_{1}=(y+z) / 4$ and $w_{2}=(y-4 z) / 4$, in (2.1) we get

$$
f\left(w_{1}\right)+f\left(w_{2}\right)=f\left(w_{1}+w_{2}\right)
$$

for all $w_{1}, w_{2} \in S$. Hence, $f$ is additive.

Theorem 2.2. Let $\varphi: S \times S \times S \rightarrow \mathbb{R}^{+} \cup\{0\}$ be a function such that

$$
\lim _{n \rightarrow \infty} \max \left[\frac{\varphi\left(2^{n} x, 2^{n} y, 2^{n} z\right)}{|2|^{n}}, \frac{\varphi\left(-2^{n} x,-2^{n} y,-2^{n} z\right)}{|2|^{n}}\right]=0
$$


for all $x, y, z \in S$ and let the limit

$$
\tilde{\varphi}(z):=\lim _{n \rightarrow \infty} \max \left\{\max \left[\frac{\varphi\left(0,2.2^{i} z, 2^{i} z\right)}{|2|^{i}}, \frac{\varphi\left(0,-2.2^{i} z,-2^{i} z\right)}{|2|^{i}}\right] ; 0 \leq i<n\right\}
$$

exists for all $z \in S$. Suppose that $f: S \rightarrow X$ with $f(0)=0$ is a mapping satisfying

$$
\left\|f\left(\frac{x+y+z}{4}\right)+f\left(\frac{3 x-y-4 z}{4}\right)+f\left(\frac{4 x+3 z}{4}\right)\right\| \leq|2|\|f(x)\|+\varphi(x, y, z)
$$

for all $x, y, z \in S$. Then there exists an additive mapping $h: S \rightarrow X$ such that

$$
\left\|\frac{f(z)-f(-z)}{2}-h(z)\right\| \leq \frac{1}{|2|^{2}} \tilde{\varphi}(z)
$$

for all $z \in S$. Moreover, if

$$
\lim _{k \rightarrow \infty} \lim _{n \rightarrow \infty} \max \left\{\max \left[\frac{\varphi\left(0,2 \cdot 2^{i} z, 2^{i} z\right)}{|2|^{i}}, \frac{\varphi\left(0,-2 \cdot 2^{i} z,-2^{i} z\right)}{|2|^{i}}\right] ; k \leq i<n+k\right\}=0
$$

then $h$ is the unique additive mapping satisfying (2.9).

Proof. Putting $x=0$ and $y=2 z$ in (2.8), we get

$$
\left\|2 f\left(\frac{3 z}{4}\right)+f\left(\frac{-3 z}{2}\right)\right\| \leq \varphi(0,2 z, z)
$$

for all $z \in S$. Replacing $z$ by $4 z / 3$ in (2.11), we obtain

$$
\|2 f(z)+f(-2 z)\| \leq \varphi\left(0, \frac{8 z}{3}, \frac{4 z}{3}\right)
$$

for all $z \in S$. Replacing $z$ by $-z$ in (2.12), we get

$$
\|2 f(-z)+f(2 z)\| \leq \varphi\left(0, \frac{-8 z}{3}, \frac{-4 z}{3}\right)
$$

for all $z \in S$. Let $g(z):=(f(z)-f(-z)) / 2$. It follows from (2.12) and (2.13) that

$$
\|g(2 z)-2 g(z)\| \leq \frac{1}{|2|} \max \left[\varphi\left(0, \frac{8 z}{3}, \frac{4 z}{3}\right), \varphi\left(0, \frac{-8 z}{3}, \frac{-4 z}{3}\right)\right]
$$


for all $z \in S$. Replacing $z$ by $2^{n-1} z$ in (2.14), we get

$$
\left\|\frac{g\left(2^{n} z\right)}{2^{n}}-\frac{g\left(2^{n-1} z\right)}{2^{n-1}}\right\| \leq \frac{1}{|2|^{n+1}} \max \left[\varphi\left(0, \frac{8.2^{n-1} z}{3}, \frac{4.2^{n-1} z}{3}\right), \varphi\left(0, \frac{-8.2^{n-1} z}{3}, \frac{-4.2^{n-1} z}{3}\right)\right]
$$

for all $z \in S$. It follows from (2.6) and (2.15) that the sequence $\left\{g\left(2^{n} z\right) / 2^{n}\right\}$ is Cauchy. Since $X$ is complete, we conclude that $\left\{g\left(2^{n} z\right) / 2^{n}\right\}$ is convergent. Set $h(z):=\lim _{n \rightarrow \infty}\left(g\left(2^{n} z\right) / 2^{n}\right)$ for all $z \in S$. Using induction one can show that

$$
\begin{aligned}
& \left\|\frac{g\left(2^{n} z\right)}{2^{n}}-g(z)\right\| \\
& \quad \leq \frac{1}{|2|^{2}} \max \left\{\max \left[\frac{\varphi\left(0,8.2^{k} z / 3,4.2^{k} z / 3\right)}{|2|^{k}}, \frac{\varphi\left(0,-8.2^{k} z / 3,-4.2^{k} z / 3\right)}{|2|^{k}}\right] ; 0 \leq k<n\right\}
\end{aligned}
$$

for all $z \in S$ and $n \in \mathbb{N}$. By taking $n$ to approach infinity in (2.16) and using (2.7) one obtains (2.9).

It follows from (2.8) that

$$
\begin{aligned}
& \left\|h\left(\frac{x+y+z}{4}\right)+h\left(\frac{3 x-y-4 z}{4}\right)+h\left(\frac{4 x+3 z}{4}\right)\right\| \\
& =\lim _{n \rightarrow \infty} \frac{1}{|2|^{n}}\left\|g\left(2^{n}\left(\frac{x+y+z}{4}\right)\right)+g\left(2^{n}\left(\frac{3 x-y-4 z}{4}\right)\right)+g\left(2^{n}\left(\frac{4 x+3 z}{4}\right)\right)\right\| \\
& =\lim _{n \rightarrow \infty} \frac{1}{|2|^{n+1}} \| \frac{f\left(2^{n}(x+y+z)\right)}{4}+\frac{f\left(2^{n}(3 x-y-4 z)\right)}{4}+\frac{f\left(2^{n}(4 x+3 z)\right)}{4} \\
& \quad-\frac{f\left(2^{n}(-x-y-z)\right)}{4}-\frac{f\left(2^{n}(-3 x+y+4 z)\right)}{4}-\frac{f\left(2^{n}(-4 x-3 z)\right)}{4} \| \\
& \leq \frac{1}{|2|^{n}}\left\|f\left(2^{n} z\right)-f\left(-2^{n} z\right)\right\|+\lim _{n \rightarrow \infty} \frac{1}{|2|} \max \left[\frac{\varphi\left(2^{n} x, 2^{n} y, 2^{n} z\right)}{|2|^{n}}, \frac{\varphi\left(-2^{n} x,-2^{n} y,-2^{n} z\right)}{|2|^{n}}\right] \\
& =|2|\|h(z)\|
\end{aligned}
$$

for all $x, y, z \in S$. So,

$$
\left\|h\left(\frac{x+y+z}{4}\right)+h\left(\frac{3 x-y-4 z}{4}\right)+h\left(\frac{4 x+3 z}{4}\right)\right\| \leq|2|\|h(z)\|
$$

for all $x, y, z \in S$. By Lemma 2.1, the mapping $h: S \rightarrow X$ is additive. 
Now, let $T: S \rightarrow X$ be another additive mapping satisfying (2.9). Then we have

$$
\begin{aligned}
\|h(z)-T(z)\| & =\frac{1}{|2|^{k}}\left\|h\left(2^{k} z\right)-T\left(2^{k} z\right)\right\| \\
& \leq \frac{1}{|2|^{k}} \max \left[\left\|T\left(2^{k} z\right)-g\left(2^{k} z\right)\right\|,\left\|g\left(2^{k} z\right)-h\left(2^{k} z\right)\right\|\right] \\
& \leq \frac{1}{|2|^{2}} \lim _{k \rightarrow \infty} \lim _{n \rightarrow \infty} \max \left\{\max \left[\frac{\varphi\left(0,2.2^{i} z, 2^{i} z\right)}{|2|^{i}}, \frac{\varphi\left(0,-2.2^{i} z,-2^{i} z\right)}{|2|^{i}}\right] ; k \leq i<i+k\right\} \\
& =0
\end{aligned}
$$

for all $z \in S$. Therefore $h=T$. This completes the proof of the uniqueness of $h$.

Corollary 2.3. Let $p>1$ and $\theta$ be positive real numbers, and let $f: S \rightarrow X$ be a mapping satisfying

$$
\left\|f\left(\frac{x+y+z}{4}\right)+f\left(\frac{3 x-y-4 z}{4}\right)+f\left(\frac{4 x+3 z}{4}\right)\right\| \leq|2|\|f(x)\|+\theta\left(\|x\|^{p}+\|y\|^{p}+\|z\|^{p}\right)
$$

for all $x, y, z \in S$. If $|2|<1$ then there exists a unique additive mapping $h: S \rightarrow X$ such that

$$
\left\|\frac{f(z)-f(-z)}{2}-h(z)\right\| \leq \frac{2 \theta}{|2|^{2}}\|z\|^{p}
$$

for all $z \in S$.

Proof. Defining $\varphi: S \times S \times S \rightarrow X$ by $\varphi(x, y, z):=\theta\left(\|x\|^{p}+\|y\|^{p}+\|z\|^{p}\right)$ we have

$$
\begin{aligned}
& \lim _{n \rightarrow \infty} \max \left[\frac{\varphi\left(2^{n} x, 2^{n} y, 2^{n} z\right)}{|2|^{n}}, \frac{\varphi\left(-2^{n} x,-2^{n} y,-2^{n} z\right)}{|2|^{n}}\right] \\
& =\lim _{n \rightarrow \infty} \frac{\theta|2|^{n p}}{|2|^{n}}\left(\|x\|^{p}+\|y\|^{p}+\|z\|^{p}\right)=0, \\
& \tilde{\varphi}(z):=\lim _{n \rightarrow \infty} \max \left\{\max \left[\frac{\varphi\left(0,2.2^{i} z, 2^{i} z\right)}{|2|^{i}}, \frac{\varphi\left(0,-2.2^{i} z,-2^{i} z\right)}{|2|^{i}}\right] ; 0 \leq i<n\right\} \\
& =\lim _{n \rightarrow \infty} \max \left\{\frac{|2|^{(i+1) p}+|2|^{i p}}{|2|^{i}} \theta\|z\|^{p} ; 0 \leq i<n\right\} \leq 2 \theta\|z\|^{p}, \\
& \lim _{k \rightarrow \infty} \lim _{n \rightarrow \infty} \max \left\{\max \left[\frac{\varphi\left(0,2.2^{i} z, 2^{i} z\right)}{|2|^{i}}, \frac{\varphi\left(0,-2.2^{i} z,-2^{i} z\right)}{|2|^{i}}\right] ; k \leq i<n+k\right\} \\
& \leq \lim _{k \rightarrow \infty}|2|^{k p} \theta\|z\|^{p}=0
\end{aligned}
$$

for all $z \in S$.

Applying Theorem 2.2, we conclude the required result. 
Corollary 2.4. Let $\psi: \mathbb{R}^{+} \cup\{0\} \rightarrow \mathbb{R}^{+} \cup\{0\}$ be a function satisfying

$$
\psi(|2| r) \leq \psi(|2|) \psi(r) \quad(r \geq 0), \psi(|2|)<|2|
$$

Let $\theta>0$, let $S$ be a normed space and let $f: S \rightarrow X$ fulfill the inequality

$$
\begin{gathered}
\left\|f\left(\frac{x+y+z}{4}\right)+f\left(\frac{3 x-y-4 z}{4}\right)+f\left(\frac{4 x+3 z}{4}\right)\right\| \\
\leq|2|\|f(x)\|+\theta[\psi(\|x\|)+\psi(\|y\|)+\psi(\|z\|)]
\end{gathered}
$$

for all $x, y, z \in S$. Then there exists a unique additive mapping $h: S \rightarrow X$ such that

$$
\left\|\frac{f(z)-f(-z)}{2}-h(z)\right\| \leq \frac{2 \theta}{|2|^{2}} \psi(\|z\|)
$$

for all $z \in S$.

Proof. Defining $\varphi: S \times S \times S \rightarrow X$ by $\varphi(x, y, z):=\theta[\psi(\|x\|)+\psi(\|y\|)+\psi(\|z\|)]$ we have

$$
\begin{aligned}
& \lim _{n \rightarrow \infty} \max \left[\frac{\varphi\left(2^{n} x, 2^{n} y, 2^{n} z\right)}{|2|^{n}}, \frac{\varphi\left(-2^{n} x,-2^{n} y,-2^{n} z\right)}{|2|^{n}}\right] \\
& \leq \theta \lim _{n \rightarrow \infty} \max \left[\left(\frac{\psi(|2|)}{|2|}\right)^{n}(\varphi(x, y, z), \varphi(-x,-y,-z))\right]=0, \\
& \tilde{\varphi}(z):=\lim _{n \rightarrow \infty} \max \left\{\max \left[\frac{\varphi\left(0,2.2^{i} z, 2^{i} z\right)}{|2|^{i}}, \frac{\varphi\left(0,-2.2^{i} z,-2^{i} z\right)}{|2|^{i}}\right] ; 0 \leq i<n\right\} \\
& =2 \theta \psi(\|z\|), \\
& \lim _{k \rightarrow \infty} \lim _{n \rightarrow \infty} \max \left\{\max \left[\frac{\varphi\left(0,2.2^{i} z, 2^{i} z\right)}{|2|^{i}}, \frac{\varphi\left(0,-2.2^{i} z,-2^{i} z\right)}{|2|^{i}}\right] ; k \leq i<n+k\right\} \\
& \leq \lim _{k \rightarrow \infty}\left(\frac{\psi(|2|)}{|2|}\right)^{k} \psi(\|z\|)=0
\end{aligned}
$$

for all $z \in S$.

Applying Theorem 2.2, we conclude the required result.

Theorem 2.5. Let $\varphi: S \times S \times S \rightarrow \mathbb{R}^{+} \cup\{0\}$ be a function such that

$$
\lim _{n \rightarrow \infty} \max \left\{|2|^{n} \varphi\left(2^{-n} x, 2^{-n} y, 2^{-n}\right),|2|^{n} \varphi\left(-2^{-n} x,-2^{-n} y,-2^{-n} z\right)\right\}=0
$$


for all $x, y, z \in S$ and let the limit

$$
\tilde{\varphi}(z):=\lim _{n \rightarrow \infty} \max \left\{\max \left[|2|^{n} \varphi\left(0,2.2^{-i} z, 2^{-i} z\right),|2|^{n} \varphi\left(0,-2.2^{-i} z,-2^{-i} z\right)\right] ; 0 \leq i<n\right\}
$$

exist for all $z \in S$. Suppose that $f: S \rightarrow X$ with $f(0)=0$ is a mapping satisfying

$$
\left\|f\left(\frac{x+y+z}{4}\right)+f\left(\frac{3 x-y-4 z}{4}\right)+f\left(\frac{4 x+3 z}{4}\right)\right\| \leq|2|\|f(x)\|+\varphi(x, y, z)
$$

for all $x, y, z \in S$. Then there exists an additive mapping $h: S \rightarrow X$ such that

$$
\left\|\frac{f(z)-f(-z)}{2}-h(z)\right\| \leq \frac{1}{|2|} \tilde{\varphi}(z)
$$

for all $z \in S$. Moreover, if

$$
\lim _{k \rightarrow \infty} \lim _{n \rightarrow \infty} \max \left\{\max \left[|2|^{i} \varphi\left(0,2.2^{-i} z, 2^{-i} z\right),|2|^{i} \varphi\left(0,-2.2^{-i} z,-2^{-i} z\right)\right] ; k \leq i<n+k\right\}=0
$$

then $h$ is the unique additive mapping satisfying (2.30).

Proof. It follows from (2.14) that

$$
\left\|2 g\left(\frac{z}{2}\right)-g(z)\right\| \leq \frac{1}{|2|} \max \left\{\varphi\left(0, \frac{4 z}{3}, \frac{2 z}{3}\right), \varphi\left(0, \frac{-4 z}{3}, \frac{-2 z}{3}\right)\right\}
$$

for all $z \in S$. Hence,

$$
\left\|2^{n} g\left(2^{-n} z\right)-2^{(n+1)} g\left(2^{-(n+1) z}\right)\right\| \leq|2|^{n} \max \left\{\varphi\left(0, \frac{4 \cdot 2^{-n} z}{3}, \frac{2 \cdot 2^{-n} z}{3}\right), \varphi\left(0, \frac{-4.2^{-n} z}{3}, \frac{-2.2^{-n} z}{3}\right)\right\}
$$

for all $z \in S$. It follows from (2.27) and (2.33) that the sequence $\left\{2^{n} g\left(2^{-n} z\right)\right\}$ is a Cauchy sequence for all $z \in S$. Since $X$ is complete, the sequence $\left\{2^{n} g\left(2^{-n} z\right)\right\}$ converges. So, one can define the mapping $h: S \rightarrow X$ by $h(z):=\lim _{n \rightarrow \infty}\left\{2^{n} g\left(2^{-n} z\right)\right\}$ for all $z \in S$.

The rest of the proof is similar to the proof of Theorem 2.2.

Remark 2.6. We can obtain similar results to Corollary 2.3 for $p<1$ and Corollary 2.4.

\section{Stability of Functional Inequality (1.13)}

We prove the generalized Hyers-Ulam stability of the functional inequality (1.13). Throughout this section, we assume that $S$ is an additive semigroup and $X$ is a complete nonArchimedean space.

We need the following lemma in the main results. 
Lemma 3.1. Let $f: S \rightarrow X$ be a mapping such that

$$
\left\|f\left(\frac{y-x}{3}\right)+f\left(\frac{x-3 z}{3}\right)+f\left(\frac{3 x+3 z-y}{3}\right)\right\| \leq\|f(x)\|
$$

for all $x, y, z \in S$. If $f(0)=0$, then the mapping $f$ is Cauchy additive.

Proof. Letting $x=y=0$ in (3.1), we get

$$
\|f(-z)+f(z)\| \leq\|f(0)\|=0
$$

for all $z \in S$. Hence, $f(-z)=-f(z)$ for all $z \in S$. Letting $x=0$ and $y=6 z$ in (3.1), we get

$$
\|f(2 z)-2 f(z)\| \leq\|f(0)\|=0
$$

for all $z \in S$. Hence,

$$
f(2 z)=2 f(z)
$$

for all $z \in S$. Letting $x=0$ and $y=9 z$ in (3.1), we get

$$
\|f(3 z)-f(z)-2 f(z)\| \leq\|f(0)\|=0
$$

for all $z \in S$. Hence,

$$
f(3 z)=3 f(z)
$$

for all $z \in S$. Letting $x=0$ in (3.1), we get

$$
\left\|f\left(\frac{y}{3}\right)+f(-z)+f\left(z-\frac{y}{3}\right)\right\| \leq\|f(0)\|=0
$$

for all $x, y, z \in S$. So,

$$
f\left(\frac{y}{3}\right)+f(-z)+f\left(z-\frac{y}{3}\right)=0
$$

for all $x, y, z \in S$. Let $t_{1}=z-y / 3$ and $t_{2}=y / 3$ in (3.8). Then

$$
f\left(t_{2}\right)-f\left(t_{1}+t_{2}\right)+f\left(t_{1}\right)=0
$$

for all $t_{1}, t_{2} \in S$. So, $f$ is additive. 
Theorem 3.2. Let $\varphi: S \times S \times S \rightarrow[0, \infty)$ be a function such that

$$
\lim _{n \rightarrow \infty} \frac{\varphi\left(2^{n} x, 2^{n} y, 2^{n} z\right)}{|2|^{n}}=0
$$

for all $x, y, z \in S$ and let the limit

$$
\tilde{\varphi}(z):=\lim _{n \rightarrow \infty} \max \left\{\max \left[\frac{\varphi\left(0,6.2^{i} z, 2^{i} z\right)}{|2|^{i}}, \frac{|2| \varphi\left(0,3.2^{i} z, 2^{i} z\right)}{|2|^{i}}\right] ; 0 \leq i<n\right\}
$$

exist for all $z \in S$. Suppose that $f: S \rightarrow X$ with $f(0)=0$ is a mapping satisfying

$$
\left\|f\left(\frac{y-x}{3}\right)+f\left(\frac{x-3 z}{3}\right)+f\left(\frac{3 x+3 z-y}{3}\right)\right\| \leq\|f(x)\|+\varphi(x, y, z)
$$

for all $x, y, z \in S$. Then there exists an additive mapping $T: S \rightarrow X$ such that

$$
\|f(z)-T(z)\| \leq \frac{1}{|2|} \tilde{\varphi}(z)
$$

for all $z \in S$. Moreover, if

$$
\lim _{k \rightarrow \infty} \lim _{n \rightarrow \infty} \max \left\{\max \left[\frac{\varphi\left(0,6.2^{i} z, 2^{i} z\right)}{|2|^{i}}, \frac{|2| \varphi\left(0,3.2^{i} z, 2^{i} z\right)}{|2|^{i}}\right] ; k \leq i<n+k\right\}=0
$$

then $T$ is the unique additive mapping satisfying (3.13).

Proof. Letting $x=0$ and $y=6 z$ in (3.12), we get

$$
\|f(2 z)+2 f(-z)\| \leq \varphi(0,6 z, z)
$$

for all $z \in S$. Putting $x=0$ and $y=3 z$ in (3.12), we get

$$
\|2 f(z)+2 f(-z)\| \leq|2| \varphi(0,3 z, z) \leq \varphi(0,3 z, z)
$$

for all $z \in S$. It follows from (3.15) and (3.16) that

$$
\|f(2 z)-2 f(z)\| \leq \max \{\varphi(0,6 z, z), \varphi(0,3 z, z)\}
$$

for all $z \in S$. Replacing $z$ by $2^{n-1} z$ in (3.17), we get

$$
\left\|\frac{f\left(2^{n} z\right)}{2^{n}}-\frac{f\left(2^{n-1} z\right)}{2^{n-1}}\right\| \leq \frac{1}{|2|^{n}} \max \left[\varphi\left(0,6.2^{n-1} z, 2^{n-1} z\right), \varphi\left(0,3.2^{n-1} z, 2^{n-1} z\right)\right]
$$


for all $z \in S$. It follows from (3.10) and (3.18) that the sequence $\left\{f\left(2^{n} z\right) / 2^{n}\right\}$ is Cauchy. Since $X$ is complete, we conclude that $\left\{f\left(2^{n} z\right) / 2^{n}\right\}$ is convergent. Set $T(z):=\lim _{n \rightarrow \infty}\left(f\left(2^{n} z\right) / 2^{n}\right)$ for all $z \in S$. Using induction one can show that

$$
\left\|\frac{f\left(2^{n} z\right)}{2^{n}}-f(z)\right\| \leq \frac{1}{|2|} \max \left\{\max \left[\frac{\varphi\left(0,6.2^{k} z, 2^{k} z\right)}{|2|^{k}}, \frac{|2| \varphi\left(0,3.2^{k} z, 2^{k} z\right)}{|2|^{k}}\right] ; 0 \leq k<n\right\}
$$

for all $z \in S$ and $n \in \mathbb{N}$. By taking $n$ to approach infinity in (3.19) and using (3.11) one obtains (3.13).

Replacing $x, y$, and $z$ by $2^{n} x, 2^{n} y$, and $2^{n} z$, respectively, in (3.12) we get

$$
\begin{aligned}
& \left\|f\left(\frac{2^{n}(y-x)}{3.2^{n}}\right)+f\left(\frac{2^{n}(x-3 z)}{3.2^{n}}\right)+f\left(\frac{2^{n}(3 x+3 z-y)}{3.2^{n}}\right)\right\| \\
& \quad \leq\left\|\frac{f\left(2^{n} x\right)}{2^{n}}\right\|+\frac{1}{|2|^{n}} \varphi\left(2^{n} x, 2^{n} y, 2^{n} z\right)
\end{aligned}
$$

for all $x, y, z \in S$. Taking the limit as $n \rightarrow \inf t y$ and using (3.10) we get

$$
\left\|T\left(\frac{y-x}{3}\right)+T\left(\frac{x-3 z}{3}\right)+T\left(\frac{3 x+3 z-y}{3}\right)\right\| \leq\|T(x)\|
$$

for all $x, y, z \in S$. By Lemma 3.1, the mapping $T: S \rightarrow X$ is additive.

If $T^{\prime}$ is another additive mapping satisfying (3.13), then

$$
\begin{aligned}
\left\|T(z)-T^{\prime}(z)\right\| & =\frac{1}{|2|^{k}}\left\|T\left(2^{k} z\right)-T^{\prime}\left(2^{k} z\right)\right\| \\
& \leq \frac{1}{|2|^{k}} \max \left[\left\|T\left(2^{k} z\right)-f\left(2^{k} z\right)\right\|,\left\|f\left(2^{k} z\right)-T^{\prime}\left(2^{k} z\right)\right\|\right] \\
& \leq \frac{1}{|2|} \lim _{k \rightarrow \infty} \lim _{n \rightarrow \infty} \max \left\{\max \left[\frac{\varphi\left(0,6.2^{i} z, 2^{i} z\right)}{|2|^{i}}, \frac{|2| \varphi\left(0,3.2^{i} z, 2^{i} z\right)}{|2|^{i}}\right] ; k \leq i<i+k\right\} \\
& =0
\end{aligned}
$$

for all $z \in S$. Therefore $T^{\prime}=T$. This proves the uniqueness of $T$. Hence, the mapping $T: S \rightarrow$ $X$ is a unique additive mapping satisfying (3.13).

Corollary 3.3. Let $p>1$ and $\theta$ be positive real numbers, and let $f: S \rightarrow X$ be a mapping satisfying

$$
\left\|f\left(\frac{y-x}{3}\right)+f\left(\frac{x-3 z}{3}\right)+f\left(\frac{3 x+3 z-y}{3}\right)\right\| \leq\|f(x)\|+\theta\left(\|x\|^{p}+\|y\|^{p}+\|z\|^{p}\right)
$$


for all $x, y, z \in S$. If $|2|<1$ then there exists a unique additive mapping $T: S \rightarrow X$ such that

$$
\|f(z)-T(z)\| \leq \frac{2 \theta}{|2|}\|z\|^{p}
$$

for all $z \in S$.

Proof. Letting $\varphi(x, y, z):=\theta\left(\|x\|^{p}+\|y\|^{p}+\|z\|^{p}\right)$ in Theorem 3.2, we obtain the result.

Corollary 3.4. Let $\psi:[0, \infty) \rightarrow[0, \infty)$ be a function satisfying

$$
\psi(|2| r) \leq \psi(|2|) \psi(r) \quad(r \geq 0), \psi(|2|)<|2| .
$$

Let $\theta>0$, let $S$ be a normed space, and let $f: S \rightarrow X$ fulfill the inequality

$$
\left\|f\left(\frac{y-x}{3}\right)+f\left(\frac{x-3 z}{3}\right)+f\left(\frac{3 x+3 z-y}{3}\right)\right\| \leq\|f(x)\|+\theta[\psi(\|x\|)+\psi(\|y\|)+\psi(\|z\|)]
$$

for all $x, y, z \in S$. Then there exists a unique additive mapping $T: S \rightarrow X$ such that

$$
\|f(z)-T(z)\| \leq \frac{2 \theta}{|2|} \psi(\|z\|)
$$

for all $z \in S$.

Proof. If we define $\varphi(x, y, z):=\theta[\psi(\|x\|)+\psi(\|y\|)+\psi(\|z\|)]$ in Theorem 3.2, then we get the result.

Remark 3.5. We can formulate a similar statement to Theorem 3.2 in which we can define the sequence $T(z):=\lim _{n \rightarrow \infty} 2^{n} f\left(z / 2^{n}\right)$ under suitable conditions on the function $\varphi$ and $\psi$ and then obtain similar results to Corollary 3.3 for $p<1$ and Corollary 3.4.

\section{References}

[1] S. M. Ulam, A Collection of Mathematical Problems, Interscience Tracts in Pure and Applied Mathematics, no. 8, Interscience, New York, NY, USA, 1960.

[2] D. H. Hyers, "On the stability of the linear functional equation," Proceedings of the National Academy of Sciences of the United States of America, vol. 27, pp. 222-224, 1941.

[3] Th. M. Rassias, "On the stability of the linear mapping in Banach spaces," Proceedings of the American Mathematical Society, vol. 72, no. 2, pp. 297-300, 1978.

[4] Z. Gajda, "On stability of additive mappings," International Journal of Mathematics and Mathematical Sciences, vol. 14, no. 3, pp. 431-434, 1991.

[5] M. Eshaghi Gordji and H. Khodaei, "Solution and stability of generalized mixed type cubic, quadratic and additive functional equation in quasi-Banach spaces," Nonlinear Analysis: Theory, Methods $\mathcal{E}$ Applications, vol. 71, no. 11, pp. 5629-5643, 2009.

[6] A. Grabiec, "The generalized Hyers-Ulam stability of a class of functional equations," Publicationes Mathematicae Debrecen, vol. 48, no. 3-4, pp. 217-235, 1996. 
[7] D. H. Hyers, G. Isac, and Th. M. Rassias, Stability of Functional Equations in Several Variables, Progress in Nonlinear Differential Equations and Their Applications, 34, Birkhäuser, Boston, Mass, USA, 1998.

[8] G. Isac and Th. M. Rassias, "On the Hyers-Ulam stability of $\psi$-additive mappings," Journal of Approximation Theory, vol. 72, no. 2, pp. 131-137, 1993.

[9] A. Najati, "On the stability of a quartic functional equation," Journal of Mathematical Analysis and Applications, vol. 340, no. 1, pp. 569-574, 2008.

[10] C.-G. Park, "On the stability of the orthogonally quartic functional equation," Bulletin of the Iranian Mathematical Society, vol. 31, no. 1, pp. 63-70, 2005.

[11] Th. M. Rassias, "On the stability of functional equations and a problem of Ulam," Acta Applicandae Mathematicae, vol. 62, no. 1, pp. 23-130, 2000.

[12] Th. M. Rassias, "On the stability of functional equations in Banach spaces," Journal of Mathematical Analysis and Applications, vol. 251, no. 1, pp. 264-284, 2000.

[13] L. Tan and S. Xiang, "On the Aleksandrov-Rassias problem and the Hyers-Ulam-Rassias stability problem," Banach Journal of Mathematical Analysis, vol. 1, no. 1, pp. 11-22, 2007.

[14] P. Găvruța, "A generalization of the Hyers-Ulam-Rassias stability of approximately additive mappings," Journal of Mathematical Analysis and Applications, vol. 184, no. 3, pp. 431-436, 1994.

[15] S. Czerwik, Stability of Functional Equations of Ulam-Hyers-Rassias Type, Hadronic Press, Palm Harbor, Fla, USA, 2003.

[16] S.-M. Jung, Hyers-Ulam-Rassias Stability of Functional Equations in Mathematical Analysis, Hadronic Press, Palm Harbor, Fla, USA, 2001.

[17] Th. M. Rassias, Ed., Functional Equations, Inequalities and Applications, Kluwer Academic Publishers, Dordrecht, The Netherlands, 2003.

[18] M. Eshaghi Gordji and M. B. Savadkouhi, "Stability of cubic and quartic functional equations in nonArchimedean spaces," Acta Applicandae Mathematicae, vol. 110, no. 3, pp. 1321-1329, 2010.

[19] M. Eshaghi Gordji and M. B. Savadkouhi, "Stability of a mixed type cubic-quartic functional equation in non-Archimedean spaces," Applied Mathematics Letters, vol. 23, no. 10, pp. 1198-1202, 2010.

[20] M. Eshaghi Gordji, M. B. Savadkouhi, and M. Bidkham, "Stability of a mixed type additive and quadratic functional equation in non-Archimedean spaces," Journal of Computational Analysis and Applications, vol. 12, no. 2, pp. 454-462, 2010.

[21] M. Eshaghi Gordji, H. Khodaei, and R. Khodabakhsh, "General quartic-cubic-quadratic functional equation in non-Archimedean normed spaces," Scientific Bulletin-University Politehnica of Bucharest. Series A, vol. 72, no. 3, pp. 69-84, 2010.

[22] M. Eshaghi Gordji, R. Khodabakhsh, H. Khodaei, and S. M. Jung, "AQCQ-functional equation in nonArchimedean normed spaces," Abstract and Applied Analysis, vol. 2010, Article ID 741942, 22 pages, 2010.

[23] A. Gilányi, "Eine zur Parallelogrammgleichung äquivalente Ungleichung," Aequationes Mathematicae, vol. 62, no. 3, pp. 303-309, 2001.

[24] J. Rätz, "On inequalities associated with the Jordan-von Neumann functional equation," Aequationes Mathematicae, vol. 66, no. 1-2, pp. 191-200, 2003.

[25] W. Fechner, "Stability of a functional inequality associated with the Jordan-von Neumann functional equation," Aequationes Mathematicae, vol. 71, no. 1-2, pp. 149-161, 2006.

[26] Y.-S. Cho and H.-M. Kim, "Stability of functional inequalities with Cauchy-Jensen additive mappings," Abstract and Applied Analysis, vol. 2007, Article ID 89180, p. 13, 2007. 


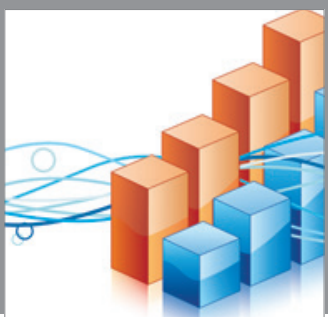

Advances in

Operations Research

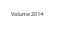

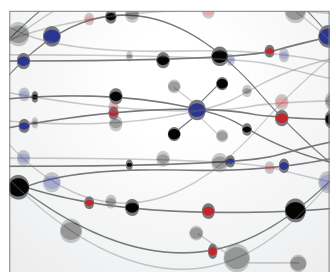

\section{The Scientific} World Journal
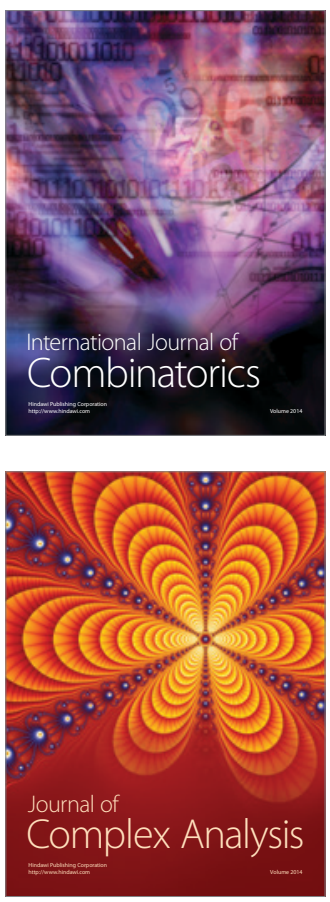

International Journal of

Mathematics and

Mathematical

Sciences
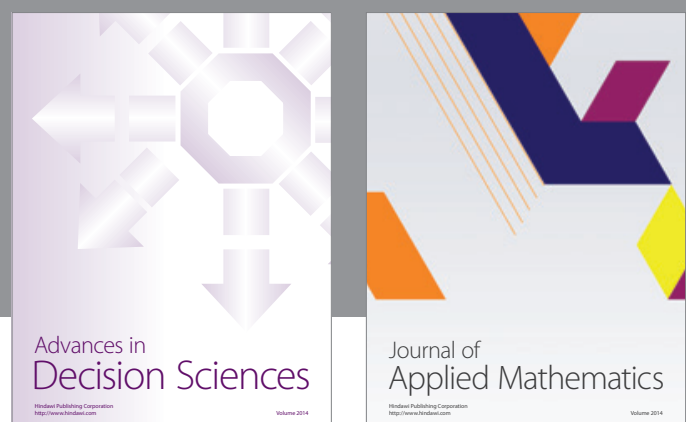

Journal of

Applied Mathematics
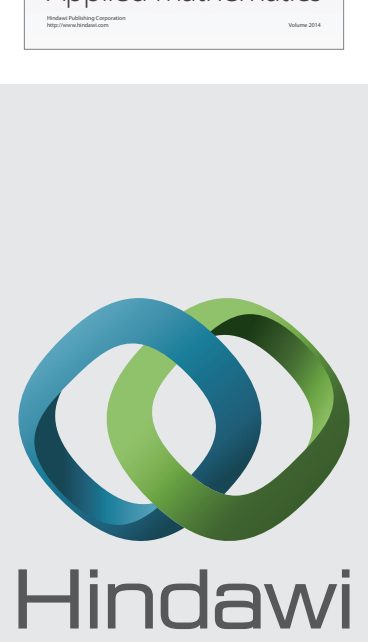

Submit your manuscripts at http://www.hindawi.com
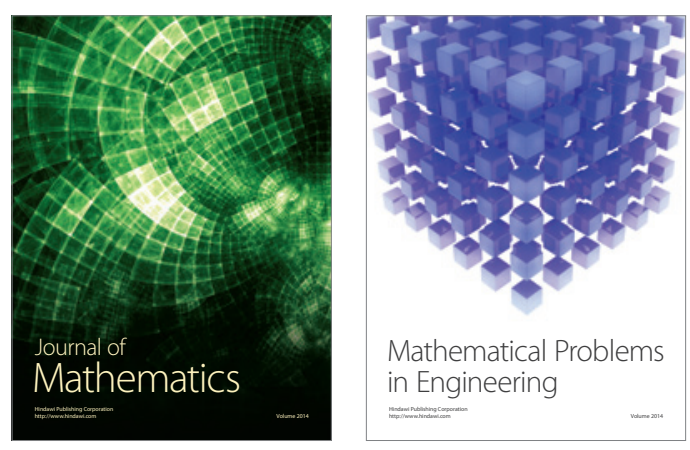

Mathematical Problems in Engineering
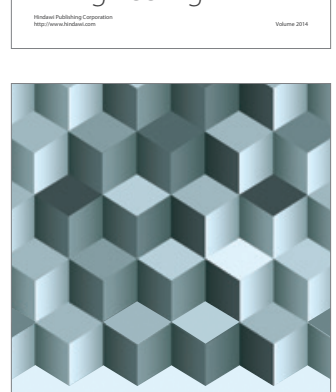

Journal of

Function Spaces
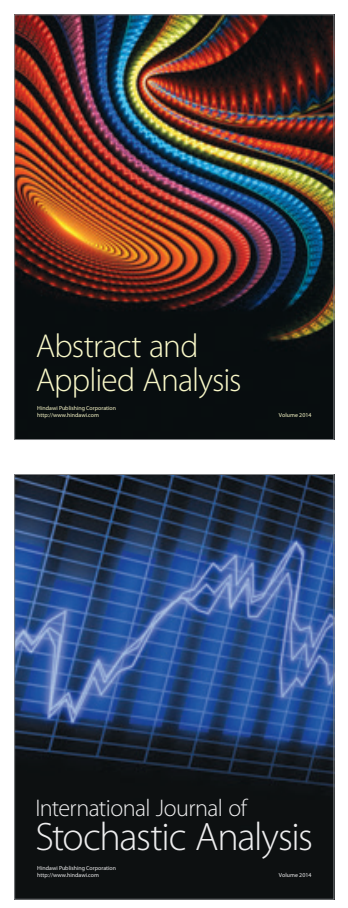

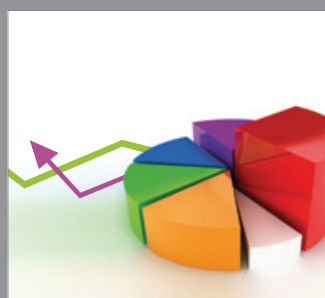

ournal of

Probability and Statistics

Promensencen
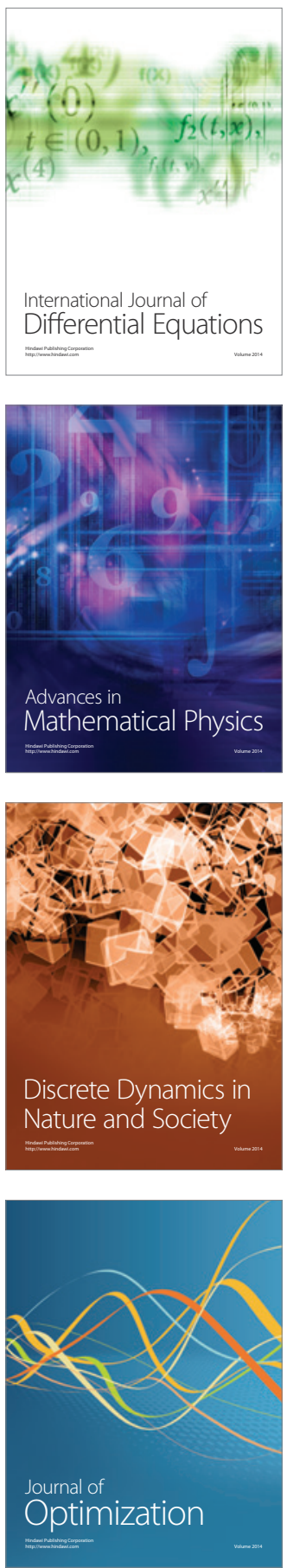